\title{
Global stabilization of feedforward systems with exponentially unstable Jacobian linearization
}

\author{
F. Grognard ${ }^{a, *}$, R. Sepulchre ${ }^{\mathrm{b}}$, G. Bastin ${ }^{\mathrm{a}}$ \\ ${ }^{a}$ Center for Systems Engineering and Applied Mechanics, Université catholique de Louvain, Av. G. Lemaitre 4, \\ B1348 Louvain-La-Neuve, Belgium \\ ${ }^{\mathrm{b}}$ Institut Montefiore, B28, Université de Liège, B4000 Liège Sart-Tilman, Belgium
}

Received 9 April 1998; received in revised form 23 November 1998; accepted 20 January 1999

\begin{abstract}
The global stabilization of a class of feedforward systems having an exponentially unstable Jacobian linearization is achieved by a high-gain feedback saturated at a low level. The control law forces the derivatives of the state variables to small values along the closed-loop trajectories. This "slow control" design is illustrated with a benchmark example and its limitations are emphasized. (c) 1999 Elsevier Science B.V. All rights reserved.
\end{abstract}

Keywords: Nonlinear control; Global stabilization; Feedforward systems

\section{Introduction}

The global stabilization of nonlinear systems has been the subject of an important literature over the last decade and significant progresses have been made towards the development of systematic design methods and a better understanding of structural limitations to large regions of attractions (see $[1,2,4,6,9,10]$ and references therein). It is fair to say that most of existing results have been obtained by exploiting certain triangularity properties of the considered system's differential equations. These structural properties can be classified in two categories and it is sufficient for our purpose to illustrate them on the system

$$
\begin{aligned}
\dot{x}_{1} & =f_{1}\left(x_{1}\right)+x_{2}+g_{1}\left(x_{2}, x_{3}, \ldots, x_{n}, u\right), \\
\dot{x}_{2} & =f_{2}\left(x_{1}, x_{2}\right)+x_{3}+g_{2}\left(x_{3}, \ldots, x_{n}, u\right), \\
& \vdots \\
\dot{x}_{n} & =f_{n}\left(x_{1}, \ldots, x_{n}\right)+u+g_{n}(u),
\end{aligned}
$$

where $x=\left(x_{1}, \ldots x_{n}\right) \in \mathbb{R}^{n}$ and $u \in \mathbb{R}$. We view (1) as a chain of integrators perturbed by feedback connections ( $f_{i}$ functions) and feedforward connections ( $g_{i}$ functions). The two possible forms of triangularity are obtained

\footnotetext{
* Corresponding author. Tel.: +32-10-478030; fax: +32-10-472180.

E-mail address: grognard@auto.ucl.ac.be (F. Grognard).
} 
by setting to zero either the feedforward terms or the feedback terms:

- Setting $g_{i} \equiv 0$ yields a system in "strict feedback form". In this case, the absence of feedforward connections puts no limitations on the available gain at the input of each integrator. Thanks to this property, systems in strict feedback form are globally stabilizable without further restrictions on the feedback nonlinearities. Even strongly destabilizing nonlinearities can be compensated for with sufficiently high gain feedback (see $[2,4,6,8,9])$.

- Setting $f_{i} \equiv 0$ yields a system in "strict feedforward form". In this case, the absence of feedback connections limits the instability of the open-loop system (in particular the Jacobian linearization can not be exponentially unstable). Thanks to this property, a low gain at the input of each integrator is sufficient to stabilize the system and systems in strict feedforward form are globally stabilizable without further restriction of the feedforward connections (except for the fact that the Jacobian linearization is required to be controllable). Even if the feedforward nonlinearities impose severe limitations on the available gain, stabilization is achieved with sufficiently low gain feedback (see [5,7,11]).

The present paper aims at a (very first) step towards design methods which could bridge the two extreme triangular classes just described, by simultaneously allowing for the presence of (destabilizing) feedback connections and ("gain limiting") feedforward connections. Our result is best illustrated with the help of the simple benchmark system

$$
\begin{aligned}
& \dot{x}_{1}=f_{1}\left(x_{1}\right)+x_{2}+g_{1}\left(x_{2}, x_{3}\right), \\
& \dot{x}_{2}=x_{3}, \\
& \dot{x}_{3}=u .
\end{aligned}
$$

Existing methods for feedforward systems require the feedback connection $f_{1}\left(x_{1}\right)$ to be missing or "stabilizing", that is $f_{1}(s) s \leqslant 0$. Existing methods for strict feedback systems require $g_{1}\left(x_{2}, x_{3}\right) \equiv 0$. Our result will show that a bounded destabilizing nonlinearity $f_{1}\left(x_{1}\right)$ can be tolerated provided that the feedforward term $g_{1}\left(x_{2}, x_{3}\right)$ is not "gain limiting" but only "rate limiting", that is, $g_{1}\left(x_{2}, x_{3}\right)=g_{1}\left(x_{3}\right)=g_{2}\left(\dot{x}_{2}\right)$, and is at least quadratic near the origin. Our design will enforce the convergence of solutions towards a region of the state space where $\dot{x}_{2}(t)$ is kept small enough, but not necessarily $x_{2}(t)$, thereby guaranteeing enough gain to compensate for the destabilizing connection $f_{1}\left(x_{1}\right)$. This design can be viewed as a slow control design in contrast to the low gain design previously considered for feedforward systems (see [5,7,11]).

Expanding on this idea, we achieve global stabilization of systems which can be written in the form

$$
\begin{gathered}
\dot{x}_{1}=f_{1}\left(x_{1}\right)+x_{2}+g_{1}\left(\dot{x}_{2}, \dot{x}_{3}, \ldots, \dot{x}_{n}\right), \\
\dot{x}_{2}=f_{2}\left(x_{2}\right)+x_{3}+g_{2}\left(\dot{x}_{3}, \ldots, \dot{x}_{n}\right), \\
\vdots \\
\dot{x}_{n-1}=f_{n-1}\left(x_{n-1}\right)+x_{n}+g_{n-1}\left(\dot{x}_{n}\right), \\
\dot{x}_{n}=f_{n}\left(x_{n}\right)+u,
\end{gathered}
$$

with $x \in \mathbb{R}^{n}, u \in \mathbb{R}$, where we have

- Feedback connections $f_{i}\left(x_{i}\right)$ which are depending only on the local integrator state. They are bounded with bounded derivatives for all $x_{i} \in \mathbb{R}$;

- Rate limiting feedforward connections $g_{i}$ with an upper triangular structure. These functions are at least quadratic near the origin.

Our design will enforce convergence of the solutions towards a sequence of nested manifolds where an increasing number of state derivatives $\dot{x}_{i}$ are kept small. Near-invariance of these manifolds will be guaranteed despite of the destabilizing feedback connections by allowing for enough gain in a restricted neighborhood of the manifolds. Section 2 of the paper describes our main result. Limitations of the present approach and its relation to other contributions is discussed in Section 4 (see $[3,12])$. 


\section{Global stabilization of feedforward systems}

The next theorem is the main result of this paper. We present a recursive design to achieve Global Asymptotic and Local Exponential Stability of the origin of system (3) using the saturation function:

$$
\text { sat } \begin{aligned}
: \mathbb{R} & \rightarrow[-1,1] \\
s & \rightarrow \operatorname{sat}(s)=\operatorname{sign}(s) \min (|s|, 1)
\end{aligned}
$$

Theorem 1. Consider the $\mathscr{C}^{1}$ system:

$$
\begin{aligned}
& \dot{x}_{1}=f_{1}\left(x_{1}\right)+x_{2}+g_{1}\left(\dot{x}_{2}, \dot{x}_{3}, \ldots, \dot{x}_{n}\right), \\
& \dot{x}_{2}=f_{2}\left(x_{2}\right)+x_{3}+g_{2}\left(\dot{x}_{3}, \ldots, \dot{x}_{n}\right), \\
& \quad \vdots \\
& \dot{x}_{n-1}=f_{n-1}\left(x_{n-1}\right)+x_{n}+g_{n-1}\left(\dot{x}_{n}\right), \\
& \dot{x}_{n}=f_{n}\left(x_{n}\right)+u,
\end{aligned}
$$

with $x_{i}, u \in \mathbb{R}$. Suppose that the feedback interconnections are bounded, with bounded derivatives, i.e. $\exists M_{i}, D_{i}>0 \forall x_{i}:\left|f_{i}\left(x_{i}\right)\right|<M_{i},\left|f_{i}^{\prime}\left(x_{i}\right)\right|<D_{i}$ and the $g_{i}$ functions are at least quadratic near the origin.

Then there exists positive constants $\varepsilon>0$ (sufficiently small) and $K>0$ (sufficiently large) such that the origin of the system is globally asymptotically and locally exponentially stabilized by

$$
u=-f_{n}\left(x_{n}\right)-\varepsilon_{n} \operatorname{sat}\left(\frac{K_{n} e_{n}}{\varepsilon_{n}}\right)
$$

where

$$
\begin{aligned}
& e_{1}=x_{1}, \\
& e_{i}=x_{i}+f_{i-1}\left(x_{i-1}\right)+\varepsilon_{i-1} \operatorname{sat}\left(\frac{K_{i-1} e_{i-1}}{\varepsilon_{i-1}}\right) \quad \text { for } i \in\{2, \ldots, n\}
\end{aligned}
$$

for a suitable choice of $\varepsilon_{i}$ and $K_{i}$. One such choice is

$$
K_{1}=K, \quad K_{i+1}=16\left(K_{i}+D_{i}\right), \quad \varepsilon_{1}=\varepsilon, \quad \frac{\varepsilon \prod_{j=2}^{i} K_{j}}{2^{i-1}} \leqslant \varepsilon_{i} \leqslant \frac{3}{2} \frac{\varepsilon \prod_{j=2}^{i} K_{j}}{2^{i-1}} .
$$

Proof. Consider the nested sequence of subsets $\Omega_{1} \subset \cdots \subset \Omega_{n} \subset \mathbb{R}^{n}$ defined by

$$
\Omega_{i}=\left\{x \in \mathbb{R}^{n}|| e_{j} \mid \leqslant \varepsilon_{j} / K_{j}, j \geqslant i\right\}
$$

whose choice is suggested by the form of the system in $e$ coordinates:

$$
\begin{aligned}
\dot{e}_{1}= & e_{2}-\varepsilon_{1} \operatorname{sat}\left(\frac{K_{1} e_{1}}{\varepsilon_{1}}\right)+g_{1}\left(\dot{x}_{2}, \dot{x}_{3}, \ldots, \dot{x}_{n}\right), \\
\vdots & \\
\dot{e}_{k}= & e_{k+1}-\varepsilon_{k} \operatorname{sat}\left(\frac{K_{k} e_{k}}{\varepsilon_{k}}\right)+\frac{\mathrm{d}_{k-1}}{\mathrm{~d} x_{k-1}}\left(e_{k}-\varepsilon_{k-1} \operatorname{sat}\left(\frac{K_{k-1} e_{k-1}}{\varepsilon_{k-1}}\right)+g_{k-1}\left(\dot{x}_{k}, \ldots, \dot{x}_{n}\right)\right) \\
& +g_{k}\left(\dot{x}_{k+1}, \ldots, \dot{x}_{n}\right)+\frac{\mathrm{d} \varepsilon_{k-1} \operatorname{sat}\left(\frac{K_{k-1} e_{k-1}}{\varepsilon_{k-1}}\right)}{\mathrm{d} e_{k-1}} \dot{e}_{k-1}, \\
& \vdots \\
\dot{e}_{n}= & -\varepsilon_{n} \operatorname{sat}\left(\frac{K_{n} e_{n}}{\varepsilon_{n}}\right)+\frac{\mathrm{d}_{n-1}}{\mathrm{~d} x_{n-1}}\left(e_{n}-\varepsilon_{n-1} \operatorname{sat}\left(\frac{K_{n-1} e_{n-1}}{\varepsilon_{n-1}}\right)+g_{n-1}\left(\dot{x}_{n}\right)\right)+\frac{\mathrm{d} \varepsilon_{n-1} \operatorname{sat}\left(\frac{K_{n-1} e_{n-1}}{\varepsilon_{n-1}}\right)}{\mathrm{d} e_{n-1}} \dot{e}_{n-1} .
\end{aligned}
$$


We will show that choice (4) for the parameters $K_{i}$ and $\varepsilon_{i}$ ensures the following properties:

(i) each $\Omega_{i}$ is invariant,

(ii) each solution reaches $\Omega=\Omega_{1}$ in finite time,

(iii) each solution in $\Omega$ converges exponentially to the equilibrium $x=0$.

We first show that the Jacobian linearization of the closed-loop system is Hurwitz when the parameters $K_{i}$ are chosen according to (4), with $K$ large enough. The Jacobian linearization of the system in $x$ coordinates is:

$$
\left\{\begin{aligned}
\dot{x}_{1} & =F_{1} x_{1}+x_{2} \\
& \vdots \\
\dot{x}_{i} & =F_{i} x_{i}+x_{i+1} \\
& \vdots \\
\dot{x}_{n} & =-\sum_{k=1}^{n}\left(\prod_{j=k+1}^{n} K_{j}\right)\left(F_{k}+K_{k}\right) x_{k}
\end{aligned}\right.
$$

where we define $F_{i}=f_{i}^{\prime}(0)$ (with $F_{n}=0$ because $f_{n}(x)$ is compensated by $u$ ). Introducing the change of coordinates $y_{i}=\frac{x_{i}}{K^{i}}$ and the new time scale $s=K t$, the matrix of this system can be decomposed into two parts:

$$
\begin{aligned}
A= & \left(\begin{array}{ccccccc}
0 & 1 & 0 & \cdots & 0 & 0 \\
0 & 0 & 1 & \cdots & 0 & 0 \\
\vdots & & \vdots & & \vdots & \\
0 & 0 & 0 & \cdots & 0 & 1 \\
-a_{n} & -a_{n-1} & -a_{n-2} & & -a_{2} & -a_{1}
\end{array}\right) \\
& +\left(\begin{array}{cccccc}
\times & 0 & 0 & \cdots & 0 & 0 \\
0 & \times & 0 & \cdots & 0 & 0 \\
\vdots & \vdots & & \vdots \\
0 & 0 & 0 & \cdots & \times & 0 \\
\times & \times & \times & & \times & 0
\end{array}\right)=T+U
\end{aligned}
$$

where $a_{i}=\lim _{K \rightarrow \infty} \frac{K_{n} \cdots K_{n-i+1}}{K^{i}}$. The choice (4) ensure that the matrix $T$ is Hurwitz and that the matrix $U$ converges to 0 as $K$ tends to $\infty$. Therefore, the matrix $A$ is Hurwitz for $K$ large enough. We have thus fixed the gains $K_{i}$ such that our controller ensures local exponential stability of the system. The original system controlled without saturation has a region of attraction $\Lambda$ (independent of $\varepsilon$ ). The rest of the proof will show that any solution of the controlled system reaches the set $\Omega$ and that we can design this set to be included inside $\Lambda$ by taking $\varepsilon$ small enough.

To this end, we use the following lemma proven in appendix.

Lemma 1. Suppose that the assumptions of Theorem 1 are satisfied with the parameters chosen such that

$$
\begin{aligned}
\varepsilon_{1} & >\frac{\varepsilon_{2}}{K_{2}}, \\
\varepsilon_{i} & >\frac{\varepsilon_{i+1}}{K_{i+1}}+2\left(D_{i-1}+K_{i-1}\right) \varepsilon_{i-1} \quad i \in\{2, \ldots, n-1\}, \\
\varepsilon_{n} & >2\left(D_{n-1}+K_{n-1}\right) \varepsilon_{n-1},
\end{aligned}
$$

then the constraints are still satisfied after a $\mu$-scaling of the $\varepsilon_{i}$ parameters, that is if we replace $\varepsilon_{i}$ by $\mu \varepsilon_{i}$, and, for $\mu$ small enough, we have: $\forall e \in \Omega_{i}$ :

(a) $\left|\dot{x}_{i}\right|<2 \varepsilon_{i}$, 
(b) $\left|e_{i}\right|=\varepsilon_{i} / K_{i} \Rightarrow e_{i} \dot{e}_{i}<0$,

(c) $\left|\dot{e}_{i}\right|<2 \varepsilon_{i}$.

It is an easy calculation to verify that (6) is satisfied with the choice (4) and that a $\mu$-scaling is achieved by taking $\varepsilon$ small enough.

Part (i) of the proof of Theorem 1 is a consequence of Lemma 1(b). When a solution is on $\partial \Omega_{i}$, it satisfies $\left|e_{j}\right|=\varepsilon_{j} / K_{j}$ for some $j \geqslant i$. Due to the fact that $\Omega_{i} \subset \Omega_{j}$ for $j \geqslant i$, Lemma 1(b) implies $e_{j} \dot{e}_{j}<0$. Therefore, the solution stays inside $\Omega_{i}$.

In order to prove (ii), we will now show that any solution starting in $\mathbb{R}^{n} \backslash \Omega_{n}$ reaches $\Omega_{n}$ in finite time and that any solution in $\Omega_{i+1}$ reaches $\Omega_{i}$ in finite time. By the invariance property of $\Omega_{i}$, this means that any solution reaches $\Omega_{1}=\Omega$ in finite time.

Let us define

$$
\phi_{i}\left(x_{i}, e_{i}\right)=f_{i}\left(x_{i}\right)+\varepsilon_{i} \operatorname{sat}\left(K_{i} e_{i} / \varepsilon_{i}\right) .
$$

Let $x(0) \in \mathbb{R}^{n} \backslash \Omega_{n}$. As long as $x(t) \notin \Omega_{n}$, the solution satisfies

$$
\dot{x}_{n}=-\varepsilon_{n} \operatorname{sign}\left(x_{n}+\phi_{n-1}\left(x_{n-1}, e_{n-1}\right)\right)
$$

which means that $x_{n}$ converges towards $-\phi_{n-1}\left(x_{n-1}, e_{n-1}\right)$. Because $\phi_{n-1}$ is bounded, $x_{n}(t)$ eventually enters an interval where the saturation is no longer active. This happens when $\left|x_{n}+\phi_{n-1}\left(x_{n-1}, e_{n-1}\right)\right|=\left|e_{n}\right|=\varepsilon_{n} / K_{n}$ : the solution enters $\Omega_{n}$ in finite time.

Next we show that any solution in $\Omega_{i+1}$ reaches $\Omega_{i}$ in finite time. The solution satisfies

$$
\dot{x}_{i}=e_{i+1}-\varepsilon_{i} \operatorname{sat}\left(\frac{K_{i}\left(x_{i}+\phi_{i-1}\left(x_{i-1}, e_{i-1}\right)\right)}{\varepsilon_{i}}\right)+g_{i}\left(\dot{x}_{i+1}, \ldots, \dot{x}_{n}\right)
$$

and as long as $x_{i}(t) \in \Omega_{i+1} \backslash \Omega_{i}$, (7) can be rewritten using Lemma 1(b) as

$$
\dot{x}_{i}=e_{i+1}-\varepsilon_{i} \operatorname{sign}\left(x_{i}+\phi_{i-1}\left(x_{i-1}, e_{i-1}\right)\right)+\mathcal{O}\left(\varepsilon_{i+1}^{2}, \ldots, \varepsilon_{n}^{2}\right) .
$$

Because $\left|e_{i+1}\right| \leqslant \varepsilon_{i+1} / K_{i+1}<\varepsilon_{i}$, the second term dominates the others while $x_{i}(t) \in \Omega_{i+1} \backslash \Omega_{i}$. Hence $x_{i}(t)$ converges towards $\phi_{i-1}$ until the saturation stops being active, i.e. the solution has reached $\Omega_{i}$.

Repeating the argument for each $i$, the solution reaches $\Omega$ in finite time. Observe that a prescaling of the parameters $\varepsilon_{i}$ can be used to include $\Omega$ in an arbitrarily small compact set containing the origin (this means that practical stability is achieved for any choice of the $K_{i}$ satisfying (6)).

Part (iii) of the proof is direct from the fact that $A$ is Hurwitz. It is sufficient to take $\Omega$ to be included inside $\Lambda$, the region of attraction of the controlled system without saturation (because $\Omega$ is an invariant set for the system controlled with and without saturation). In order to do so, we take $\varepsilon$ small enough.

The proof of the previous theorem shows why we may use the term slow control: the derivatives of the variables are successively brought to small values in order to be able to neglect the values of the $g_{i}$ functions.

When $n=2$, condition (4) on the parameters reduces to $K_{2}>16\left(K_{1}+D_{1}\right)$ (in this case, it could be weakened to $\left.K_{2}>2\left(K_{1}+D_{1}\right)\right)$. We observe that the latter condition is stronger than the condition imposed for the local asymptotic stability, which only requires $K_{2}>D_{1}$. This shows that the $K_{i}$ constants must be chosen large enough not only to ensure local asymptotic stability, but also to render the designed manifolds near-invariant.

\section{Example}

We now illustrate the calculation of the parameters on a simple example. Consider the benchmark system (2) with $f$ a bounded function such that $\left\|f^{\prime}\left(x_{1}\right)\right\|_{\infty}<D$ and $g_{1}\left(x_{2}, x_{3}\right)=x_{3}^{2}=\dot{x}_{2}^{2}$ :

$$
\begin{aligned}
& \dot{x}_{1}=f_{1}\left(x_{1}\right)+x_{2}+x_{3}^{2}, \\
& \dot{x}_{2}=x_{3}, \\
& \dot{x}_{3}=u .
\end{aligned}
$$


The set of constraints (6) is

$$
\begin{aligned}
& \varepsilon_{1}>\varepsilon_{2} / K_{2}, \\
& \varepsilon_{2}>\varepsilon_{3} / K_{3}+2\left(D_{1}+K_{1}\right) \varepsilon_{1}, \\
& \varepsilon_{3}>2 K_{2} \varepsilon_{2},
\end{aligned}
$$

which could easily be solved for such a low-order system but becomes more intricate for larger dimensions. Therefore, we rather use the explicit parameter values that we gave in Theorem 1.

Let us take $\varepsilon_{1}=\varepsilon$ small enough and $K_{1}=K$ large enough. We choose the parameters according to (4) with $K_{i+1}=16\left(D_{i}+K_{i}\right)$ and $\varepsilon_{i}$ in the middle of the intervals:

$$
\begin{array}{lll}
K_{1}=K, & K_{2}=16(D+K), & K_{3}=256(D+K), \\
\varepsilon_{1}=\varepsilon, & \varepsilon_{2}=10 \varepsilon(D+K), & \varepsilon_{3}=1280 \varepsilon(D+K)^{2} .
\end{array}
$$

With such parameters, the control law is

$$
u=-1280 \varepsilon(D+K)^{2} \text { sat }\left[\frac{1}{5 \varepsilon(D+K)}\left(x_{3}+10 \varepsilon(D+K) \operatorname{sat}\left[\frac{8}{5 \varepsilon}\left(x_{2}+f_{1}\left(x_{1}\right)+\varepsilon \text { sat }\left[\frac{K x_{1}}{\varepsilon}\right]\right)\right]\right)\right] .
$$

We see that both $K_{i}$ and $\varepsilon_{i}$ parameters are increasing. Such a controller can be designed to satisfy any rate constraint on the control and on the states. $K$ must be taken sufficiently large in order to get asymptotic stability. $\varepsilon$ must be sufficiently small to be able to neglect the $\varepsilon_{2}^{2}$ term that will arise because of the $x_{3}^{2}=\dot{x}_{2}^{2}$ term.

We can justify on this example the boundedness condition of Theorem 1 imposed on the $f_{i}$ functions. Indeed, when $f_{1}: \mathbb{R} \rightarrow \mathbb{R}$ is a $\mathscr{C}^{1}$ function such that $\lim _{s \rightarrow+\infty} f(s)=+\infty$ and $\exists \varepsilon>0: \forall s \in \mathbb{R}: f_{1}^{\prime}(s)>\varepsilon$, system (8) can not be globally controlled to the origin. This follows from the calculation:

$$
\begin{aligned}
\frac{\mathrm{d}}{\mathrm{d} t}\left(f_{1}\left(x_{1}\right)+x_{2}\right) & =f_{1}^{\prime}\left(x_{1}\right)\left(f_{1}\left(x_{1}\right)+x_{2}\right)+f_{1}^{\prime}\left(x_{1}\right) x_{3}^{2}+x_{3} \\
& \geqslant f_{1}^{\prime}\left(x_{1}\right)\left(f_{1}\left(x_{1}\right)+x_{2}\right)-C \\
& \geqslant \varepsilon\left(f_{1}\left(x_{1}\right)+x_{2}\right)-C \quad \text { when } f_{1}\left(x_{1}\right)+x_{2} \geqslant 0,
\end{aligned}
$$

where we have used the fact that $f_{1}^{\prime}\left(x_{1}\right) x_{3}^{2}+x_{3}$ is bounded from below by a negative constant $-C$. If the initial condition of the system is such that $\varepsilon\left(f_{1}\left(x_{10}\right)+x_{20}\right)>C$, the quantity $f_{1}\left(x_{1}\right)+x_{2}$ will diverge to $+\infty$ regardless of the control law. It means that such initial conditions $\left(x_{10}, x_{20}\right)$ cannot be driven to the origin, even if $f_{1}$ is a simple linear function.

\section{Limitations of slow control}

The slow control design proposed in this paper applies to a restricted class of nonlinear systems which simultaneously present destabilizing feedback connections and "rate limiting" feedforward connections. The restrictions on the feedforward connections (to be "rate limiting" rather than "gain limiting") and the boundedness of the $f_{i}$ functions (and of their derivatives) have been justified by means of elementary (that is, scalar) controllability requirements.

A subtler limitation of slow control is the fact that the $f_{i}$ 's can not depend on the states $x_{1}, \ldots, x_{i-1}$ : the feedback connections are restricted to be "local" connections around each integrator. Thus our design can yield a slow control for the second order system

$$
\begin{aligned}
& \dot{x}_{1}=f_{1}\left(x_{1}\right)+x_{2}, \\
& \dot{x}_{2}=f_{2}\left(x_{1}, x_{2}\right)+u,
\end{aligned}
$$


but this does not mean that this slow control law can be "backstepped" to yield a slow control law for the augmented system

$$
\begin{aligned}
& \dot{x}_{1}=f_{1}\left(x_{1}\right)+x_{2}, \\
& \dot{x}_{2}=f_{2}\left(x_{1}, x_{2}\right)+x_{3}, \\
& \dot{x}_{3}=u,
\end{aligned}
$$

even if boundedness is assumed for $f_{2}$ and its derivatives. This is in contrast with recent bounded backstepping results $[3,12]$ which show that a bounded control law with bounded rate can be backstepped to yield a new bounded control law with bounded rate (when all the nonlinearities are bounded with bounded derivatives). The difference with our result is that the bound on the control magnitude (or rate) is not a free design parameter in $[3,12]$. In the present paper, this flexibility is needed to account for the feedforward nonlinearities. For this reason, it seems plausible that the boundedness assumptions of the present paper do not suffice to achieve global stabilization of a system as simple as

$$
\begin{aligned}
& \dot{x}_{1}=f_{1}\left(x_{1}\right)+x_{2}+g(u), \quad g(0)=0, \\
& \dot{x}_{2}=f_{2}\left(x_{1}\right)+x_{3}, \\
& \dot{x}_{3}=u,
\end{aligned}
$$

which violates the structure covered by the present paper only because $f_{2}$ depends on $x_{1}$ rather than $x_{2}$.

\section{Conclusion}

We have considered the global stabilization of a restricted class of nonlinear systems which simultaneously present "destabilizing" feedback connections and "rate limiting" feedforward connections. Under the restriction that the feedback connections are "local" connections around each integrator, a slow control design has been proposed which enforces a slow convergence towards a nested sequence of manifolds, the last of which is a stable manifold of the closed-loop system. Near invariance of the successive manifolds is achieved by allowing for enough gain in their neighborhood, yet keeping the control slow in the entire state space.

\section{Acknowledgment}

The authors wish to thank Stuart Townley for his useful comments. This paper presents research results of the Belgian Programme on Interuniversity Poles of Attraction, initiated by the Belgian State, Prime Minister's office for Science, Technology and Culture. The scientific responsibility rests with the authors. The first author is Aspirant FNRS at CESAME.

\section{Appendix A. Proof of Lemma 1}

(a) (By induction) Because $\dot{x}_{n}=-\varepsilon_{n} \operatorname{sat}\left(K_{n} e_{n} / \varepsilon_{n}\right)$ (a) holds for $i=n$.

Suppose now that it is true for $j=k+1 \cdots n$, we show that it is true for $j=k$. We know that $\left|\dot{x}_{j}\right|<2 \varepsilon_{j}$ for $j>k$ inside $\Omega_{k}$. We have

$$
\dot{x}_{k}=e_{k+1}-\varepsilon_{k} \operatorname{sat}\left(K_{k} e_{k} / \varepsilon_{k}\right)+g_{k}\left(\dot{x}_{k+1}, \ldots, \dot{x}_{n}\right) .
$$

Using the fact that $x$ is in $\Omega_{k}$ and $\left|\dot{x}_{j}\right|<2 \varepsilon_{j}$ for $j>k$, we obtain:

$$
\left|\dot{x}_{k}\right|<\varepsilon_{k+1} / K_{k+1}+\varepsilon_{k}+\mathcal{O}\left(\varepsilon_{i+1}^{2}, \ldots, \varepsilon_{n}^{2}\right) .
$$

By (6), we conclude that $\left|\dot{x}_{k}\right|<2 \varepsilon_{k}$ up to $\mu$-scaling (that is, by scaling all the $\varepsilon_{i}$ 's with $\mu$ sufficiently small, $\mathcal{O}\left(\varepsilon_{m}^{2}\right)$ becomes negligeable in front of the other terms and $\left|\dot{x}_{k}\right|<2 \varepsilon_{k}$ follows from (6)). 
To prove (b) and (c) we consider the system in the $e$ coordinates (Eq. (2.5)). We show (b) and (c) by induction. For $k=1$, we have

$$
\dot{e}_{1}=e_{2}-\varepsilon_{1} \operatorname{sat}\left(K_{1} e_{1} / \varepsilon_{1}\right)+g_{1}\left(\dot{x}_{2}, \dot{x}_{3}, \ldots, \dot{x}_{n}\right)
$$

which becomes, when $x \in \Omega$,

$$
\dot{e}_{1}=e_{2}-\varepsilon_{1} \operatorname{sign}\left(e_{1}\right)+g_{1}\left(\dot{x}_{2}, \dot{x}_{3}, \ldots, \dot{x}_{n}\right)
$$

where $g_{1}\left(\dot{x}_{2}, \dot{x}_{3}, \ldots, \dot{x}_{n}\right)=\mathcal{O}\left(\varepsilon_{2}^{2}, \ldots, \varepsilon_{n}^{2}\right)$ by using (a). From (6) and after $\mu$-scaling, we conclude that the derivative of $e_{1}$ has the sign of $-\varepsilon_{1} \operatorname{sign}\left(e_{1}\right)$ i.e. $e_{1} \dot{e}_{1}<0$ when $\left|e_{1}\right|=\varepsilon_{1} / K_{1}$. We also see that $\left|\dot{e}_{1}\right|$ is bounded by $\varepsilon_{1}+\varepsilon_{2} / K_{2}+\mathcal{O}\left(\varepsilon_{2}^{2}, \ldots, \varepsilon_{n}^{2}\right)<2 \varepsilon_{1}$ inside $\Omega_{1}$ (after $\mu$-scaling). Hence (b) and (c) hold for $i=1$.

Induction step: Suppose that (b) and (c) hold for $i=k-1$. We will show that (b) and (c) also hold for $i=k$. Consider the $e_{k}$ equation:

$$
\begin{aligned}
\dot{e}_{k}= & e_{k+1}-\varepsilon_{k} \operatorname{sat}\left(K_{k} e_{k} / \varepsilon_{k}\right)+\frac{\mathrm{d} f_{k-1}}{\mathrm{~d} x_{k-1}}\left(e_{k}-\varepsilon_{k-1} \operatorname{sat}\left(\frac{K_{k-1} e_{k-1}}{\varepsilon_{k-1}}\right)+g_{k-1}\left(\dot{x}_{k}, \ldots, \dot{x}_{n}\right)\right) \\
& +g_{k}\left(\dot{x}_{k+1}, \ldots, \dot{x}_{n}\right)+\frac{\mathrm{d} \varepsilon_{k-1} \operatorname{sat}\left(\frac{K_{k-1} e_{k-1}}{\varepsilon_{k-1}}\right)}{\mathrm{d} e_{k-1}} \dot{e}_{k-1} .
\end{aligned}
$$

When $x \in \Omega_{k}$, the last term of (A.1)

$$
\frac{\mathrm{d} \varepsilon_{k-1} \operatorname{sat}\left(\frac{K_{k-1} e_{k-1}}{\varepsilon_{k-1}}\right)}{\mathrm{d} e_{k-1}} \dot{e}_{k-1}
$$

is zero when $x \notin \Omega_{k-1}$ and it is $K_{k-1} \dot{e}_{k-1}$ when $x \in \Omega_{k-1}$, which is bounded by $2 K_{k-1} \varepsilon_{k-1}$ in this part o $\Omega_{k-1}$. Evaluating (A.1) when $\left|e_{k}\right|=\varepsilon_{k} / K_{k}$, we obtain that $\dot{e}_{k} e_{k}<0$ if

$$
\varepsilon_{k}>\frac{\varepsilon_{k+1}}{K_{k+1}}+D_{k-1}\left(\frac{\varepsilon_{k}}{K_{k}}+\varepsilon_{k-1}+\mathcal{O}\left(\varepsilon_{k}^{2}, \ldots, \varepsilon_{n}^{2}\right)\right)+\mathcal{O}\left(\varepsilon_{k+1}^{2}, \ldots, \varepsilon_{n}^{2}\right)+2 K_{k-1} \varepsilon_{k-1} .
$$

By (6), $\varepsilon_{k} / K_{k}<\varepsilon_{k-1}$ and $\varepsilon_{k+1} / K_{k+1}+2\left(D_{k-1}+K_{k-1}\right) \varepsilon_{k-1}<\varepsilon_{k}$, so that (A.2) is satisfied, i.e. $\dot{e}_{k} e_{k}<0$.

Using the bound (6), we analogously see that the bound $\left|\dot{e}_{k}\right|<2 \varepsilon_{k}$ is satisfied. The induction step proves (b) and (c) for $k \in\{2, \ldots, n-1\}$. For $k=n,(6)$ is slightly different. Inequality (A.2) becomes

$$
\varepsilon_{n}>D_{n-1}\left(\mathcal{O}\left(\varepsilon_{n}^{2}\right)+\frac{\varepsilon_{n}}{K_{n}}+\varepsilon_{n-1}\right)+2 K_{n-1} \varepsilon_{n-1}
$$

which follows from (6) up to $\mu$-scaling. This ends the proof.

\section{References}

[1] J.M. Coron, L. Praly, A. Teel, Feedback stabilization of nonlinear systems: sufficient conditions and Lyapunov and Input-Output techniques, In: A. Isidori (Ed.), Trends in Control, Springer, Berlin, 1995, pp. 293-347.

[2] R.A. Freeman, P.V. Kokotović, Robust Control of Nonlinear Systems, Birkhäuser, Boston, 1996.

[3] R. Freeman, L. Praly, Integrator backstepping for bounded controls and control rates, IEEE Trans. Automat. Control 43 (1998) $258-262$.

[4] A. Isidori, Nonlinear Control Systems, third ed., Springer, Berlin, London, 1995.

[5] M. Jankovic, R. Sepulchre, P.V. Kokotović, Global stabilization of nonlinear cascade systems, IEEE Trans. Automat. Control 41 (1996) 1723-1735.

[6] M. Krstić, I. Kanellakopoulos, P.V. Kokotović, Nonlinear and Adaptive Control Design, Wiley, New York, 1995.

[7] F. Mazenc, L. Praly, Adding an integration and global asymptotic stabilization of feedforward systems, IEEE Trans. Automat. Control 41 (1996) 1559-1578.

[8] A. Saberi, P.V. Kokotović, H.J. Sussmann, Global stabilization of partially linear composite systems, SIAM J. Control Optim. 28 (1990) 1491-1503. 
[9] R. Sepulchre, M. Jankovic, P.V. Kokotović, Constructive Nonlinear Control, Springer, Berlin, 1996.

[10] R. Sepulchre, Slow peaking and global stabilization of a perturbed chain of integrators, Proceedings 36th IEEE CDC, San Diego, 1997, pp. 3491-3496.

[11] A. Teel, Using saturation to stabilize a class of single-input partially linear composite systems, IFAC NOLCOS'92 Symposium, Bordeaux, June 1992.

[12] J. Tsinias, Input to state stability properties of nonlinear systems and applications to bounded feedback stabilization using saturation, ESSAIM: Control, Optim. Calculus Variations 2 (1997) 57-85. 